\title{
Optimized Mobile Edge Computing Framework for IoT based Medical Sensor Network Nodes
}

\author{
Dr. Jennifer S. Raj, \\ Department of ECE, \\ Gnanamani College of Technology, \\ Namakkal, India. \\ jennifer.raj@gmail.com
}

\begin{abstract}
Edge computing is a new computing paradigm that is rapidly emerging in various fields. Task completion is performed by various edge devices with distributed cloud computing in several conventional applications. Resource limitation, transmission efficiency, functionality and other edge network based circumstantial factors make this system more complex when compared to cloud computing. During cooperation between the edge devices, an instability occurs that cannot be ignored. The edge cooperative network is optimized with a novel framework proposed in this paper. This helps in improving the efficiency of edge computing tasks. The cooperation evaluation metrics are defined in the initial stage. Further, the performance of specific tasks are improved by optimizing the edge network cooperation. Real datasets obtained from elderly people and their wearable sensors is used for demonstrating the performance of the proposed framework. The extensive experimentation also helps in validating the efficiency of the proposed optimization algorithm.
\end{abstract}

Keywords: Wearable sensor, Mobile edge computing, Edge cooperative network, Optimization, Cloud Computing, Edge computing

\section{Introduction}

In industrial Internet of Things (IoT) based settings, wearable sensors act as the primary data sources. At the edge network significant power consumption takes places by a large number of wearable sensors due to their computational power [1]. Distributed deep learning, big data analytics and Internet of Vehicles (IoV) are some of the emerging fields in which the edge computing paradigm plays a major role. When compared to the traditional cloud computing schemes, in terms of privacy and security, edge computer offers improved performance [2]. Three main architectures, namely mobile edge computing (MEC), fog computing and cloudlets 
Journal of Ubiquitous Computing and Communication Technologies (UCCT) (2021)

Vol.03/ No.01

Pages: $33-42$

https://www.irojournals.com/jucct/

DOI: https://doi.org/10.36548/jucct.2021.1.004

are proposed for comparison with the edge computing paradigm. In both academia as well as the industry, considerable attention is attracted by the MEC architecture. Due to the significant advancement in quality of service (QoS) offered by edge computing, there has been a gradual transition from cloud to edge technology in the devices that perform computationally intensive task [3].

New opportunities and challenges are posed by the cooperation among the edge nodes and edge resource management due to the development of several edge computing applications [4]. Based on the edge environment instability, optimization of the edge computing architecture is to be performed by enhancing the cooperation among tasks. In this regard, several techniques are available in existing literature [5]. In the IoV framework, peer-to-peer offloading is performed by implementation of a road side unit (RSU) by promoting heuristic computing and artificial intelligence (AI) that allows caching and communication of resources within the vicinity of smart vehicles. The total network delay has been minimized in a remarkable manner with this model [6]. In multiple bit rate videos, the cooperative caching issue is addressed by various researchers. In multi-hop cooperative networks, the computational task assignment is optimized through iterative task assignment with the help of a heuristic algorithm. Figure 1 provides the complete architecture and possible applications of the MEC framework.

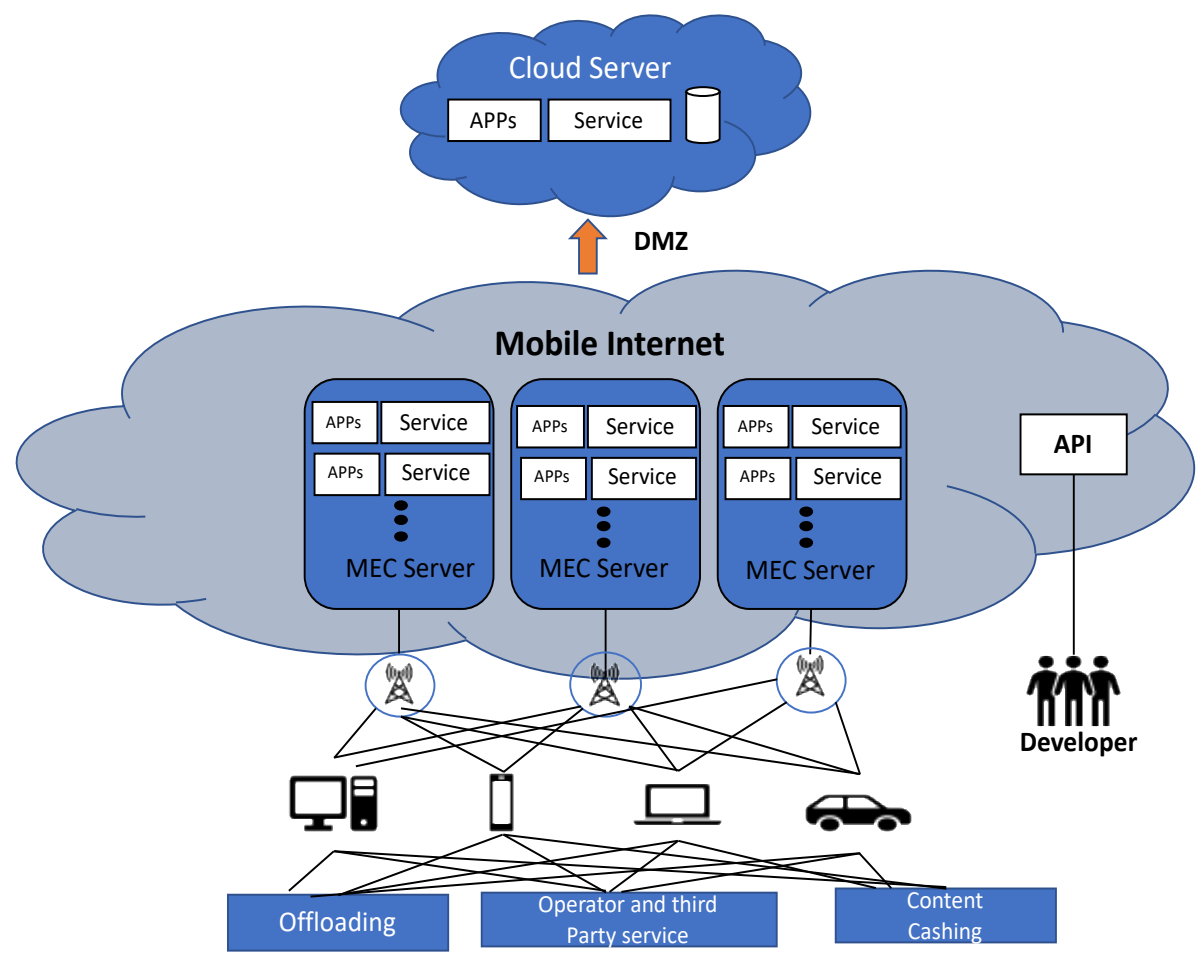

Fig. 1. MEC architecture and applications 
Journal of Ubiquitous Computing and Communication Technologies (UCCT) (2021)

Vol.03/ No.01

Pages: $33-42$

https://www.irojournals.com/jucct/

DOI: https://doi.org/10.36548/jucct.2021.1.004

\section{Related Works}

Privacy is guaranteed and response time is reduced at the edge devices by means of edge computing paradigm while exploring the potential of IoT [7]. From data producer to consumer and vice versa, the network edge is varying continuously. In mobile devices, energy saving is performed by offloading few or all computational tasks from the MEC nodes to the cloud as the computational resources are limited at terminal devices [8]. This technique is used despite the wide application of edge computing technology [9]. The edge devices and their computational capacity is exploited to the full extent in mobile phones through the face detection system. Context-aware services are provided by continuous tracking of information about the end user and making inferences consequently using edge devices by the MEC server [10]. Significant challenges are imposed on the edge nodes and devices cooperation while enriching the edge applications with huge IoT based data services by the edge computing through high quality computational capabilities.

In network analysis, the contribution of network representation technology is significant [11]. In order to gather valued data as well as to learn about the networks, network representation may be used with several edge tasks conceptualized in the networks [12]. A low dimensional vector space is mapped to a network during network representation. Further, the network nodes are represented with low dimensional dense vector [13]. In language models, sentences are simulated in the network using random walk by means of DeepWalk, thereby transforming the network representation issue into the world representation issues. Node2vec and other random walk techniques are used as a general learning tool in network embedding for subtle control of random walk [14]. The pairwise proximity of first and second order are preserved by learning network representations with Large-scale Information Network Embedding (LINE). A broader solution is provided by graph neural network (GNN) when compared with models considering only graph structures or node features [15]. A graph convolution operator termed as the bilinear GNN (BGNN) framework is used by certain researchers for augmenting the neighbor nodes representation via pairwise interactions with the corresponding weighted sum. Simplification of the algorithm is largely researched by several researchers as its complexity largely affects the output. 
Journal of Ubiquitous Computing and Communication Technologies (UCCT) (2021)

Vol.03/ No.01

Pages: $33-42$

https://www.irojournals.com/jucct/

DOI: https://doi.org/10.36548/jucct.2021.1.004

\section{Proposed Work}

ECN establishment, network representation algorithm application and low dimensional node representation are the three major stages of the proposed ECN optimization framework. The nodes and their cooperative nodes are identified initially and further, the nodes are evaluated for network optimization. The framework is expressed in figure 2. The variance in ordinary and edge network tasks must be monitored while considering the edge network tasks as the number of resources in edge network equipment is considerably small. Less than ordinary tasks dominates the computing power in edge networks. Nodes are often unique in edge cooperative tasks. The differences may be in terms of resources, computational capacity, functions and so on. Precise control of the status of all nodes and optimization is challenging due to the difference between nodes. This is analyzed with the help of a real time ECN scenario.

Abstraction of ECN and edge cooperation is performed due to the aforementioned reasons in this research. The node roles are considered for this purpose. During the nodal interaction, the nodes roles are implicit in an intuitive manner. Wearable devices and base stations are treated equally and defined as equivalent network nodes during the network establishment. The interaction records between nodes are equivalent to the tasks. Evaluation and optimization of edge cooperation has no standard technique as most of the existing literature are limited to specific task. Optimization of medical sensors on cooperative evaluation forms the basis of this motivation. Several similarities exist between the medical sensors based applications and edge computing based evaluation and optimization techniques. The characteristics that contributes towards this similarity are - limited resource availability and restricted current location adjustments, several uncertainties and unfavorable objective environment, clear overall purpose, the abilities and roles of each node varies from each other and node cooperative networks have the same data source such as IoT sensors. 




Fig. 2. Proposed ECN optimization framework

The edge tasks are used for defining the ECN. Relationship representation algorithm is explored while concentrating on the edge nodes and the cooperation relationship between them. A novel ECN optimization framework is developed using the network representation results and extraction of microoperation modes. Recommendation for replacement node and indicators for evaluation of macro cooperation may be realized. The design experiments are used for verification of the proposed indicators on data samples obtained from wearable medical sensors. The medical cooperation network is analyzed and optimized with the help of real datasets. Several edge network tasks may be applied with the proposed pervasive cooperative evaluation indicators to obtain optimal results.

\section{Results and Discussion}

Based on the wearable medical sensors of elderly persons, the medical cooperation network is selected innovatively for the purpose of experimentation and verification of our 
Journal of Ubiquitous Computing and Communication Technologies (UCCT) (2021)

Vol.03/ No.01

Pages: $33-42$

https://www.irojournals.com/jucct/

DOI: https://doi.org/10.36548/jucct.2021.1.004

framework. The real dataset selected for experimentation is introduced first. Further, comparison of the cooperation network evaluation metrics is performed with other existing models. Lastly, analysis of node replacement events that occur in the real dataset is performed. The proposed framework's efficiency is proved based on this analysis. A network evaluation indicator is used for judging the level of optimization of the ECN. The performance of optimization of the proposed framework is verified based on the change in evaluation indicator with respect to the information received by the medical sensors. ECN optimization also works towards replacing the nodes in the network with low capability of interaction. This in turn improves the overall efficiency. On obtaining the recommendation of replacement for specific nodes, actual replacement is performed and the performance of the framework is analyzed further. The difference between the actual and recommended replacement are analyzed to evaluate the efficiency of ECN optimization. Several typical cases are analyzed during experimentation in this regard. Figure 3 provides the visual data representation of the hormonal impact detected by the sensor nodes and the response to medication of two user groups based on the sensor data obtained from the wearable IoT sensors.

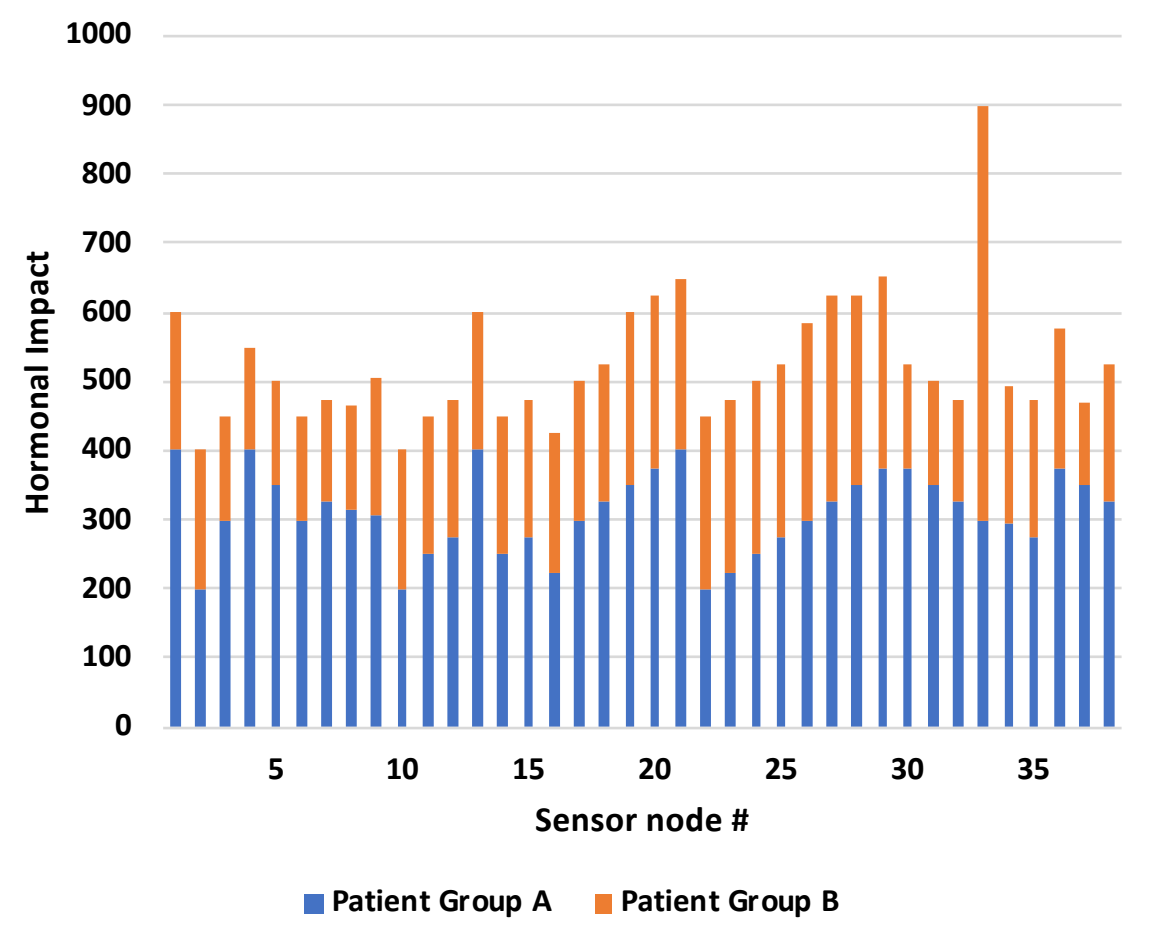

(a) 


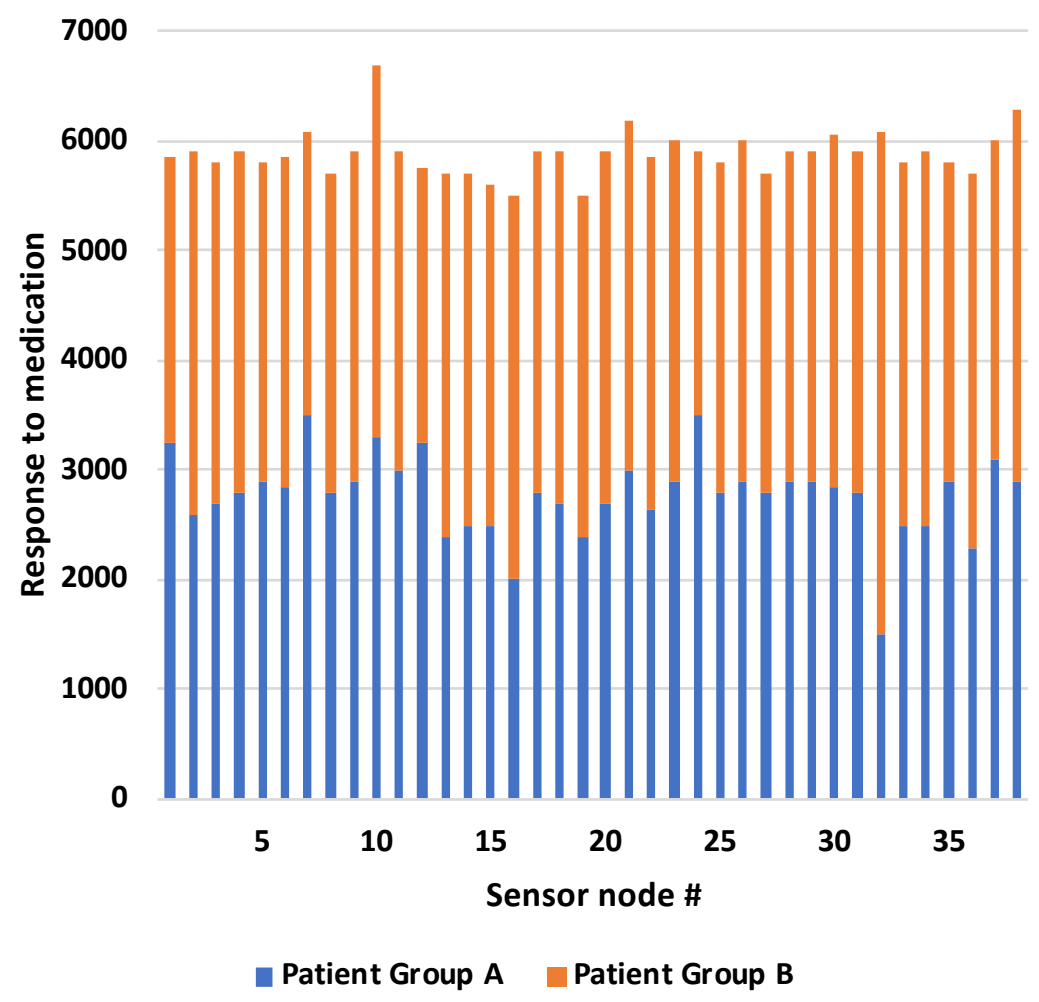

(b)

Fig. 3. Data visualization in terms of (a) Hormonal impact and (b) Response to medication for two user groups based on the wearable medical sensor data

Data samples from several wearable medical sensors in use by elderly adults are taken for the purpose of calculation of baseline metrics and QoC based cooperative evaluation indicator. Several correlation metrics are compared with the proposed framework for further testing the cooperation evaluation metrics efficiency. Correlation measurement between two different data types is measured by means of Pearson correlation. Data is correlated positively when a positive value is provided and they are correlated negatively otherwise. Correlation strength is represented by the absolute value. Between any two variables, the rank correlation may be measured using Spearman's Rank Correlation Coefficient (SRCC). Correlation between variables are evaluated with a monotonic function. When complete monotonic correlation is obtained with no duplicate values, the Spearman's correlation coefficient is +1 or -1 . Figure 4 provides the baseline and QoC indicators comparison with response to medication in terms of mean and variance. 


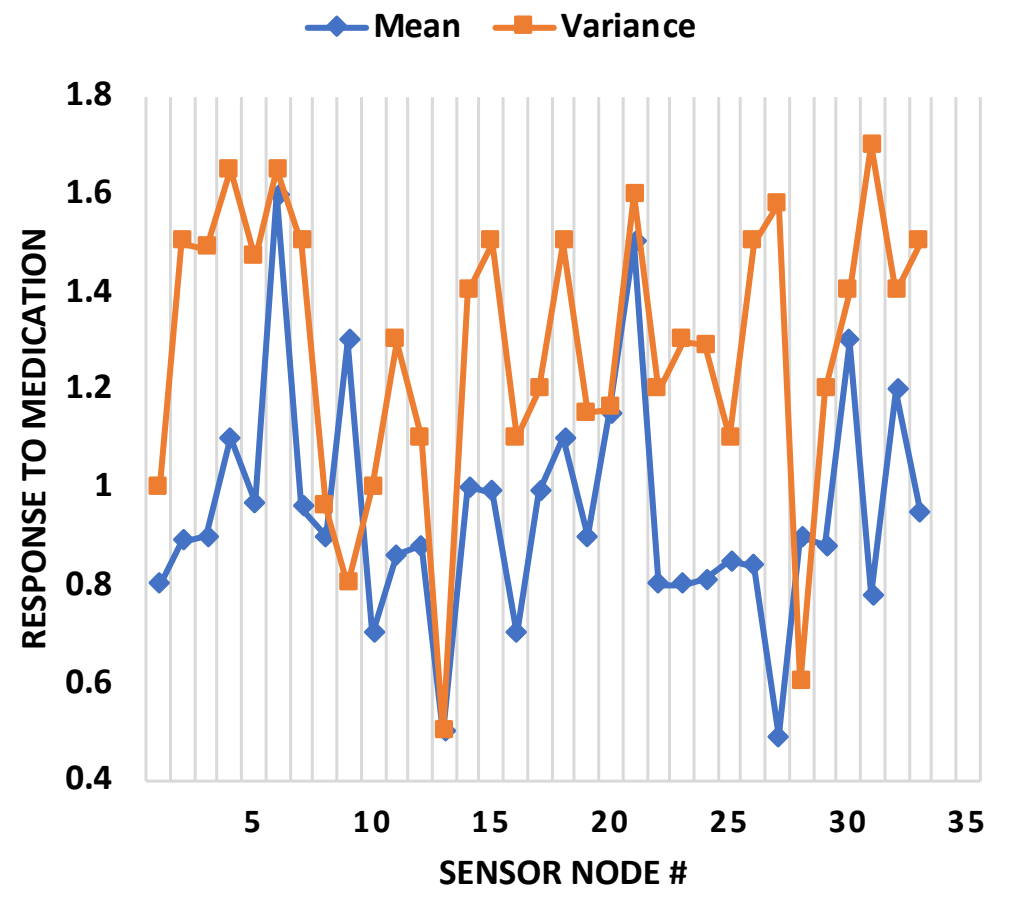

Fig. 4. Baseline and QoC indicators comparison with response to medication in terms of mean and variance

\section{Conclusion}

An optimization framework is developed for ECN in this paper. On the basis of network representation learning, the cooperative nodes extracted and the ECN definition are put forth primarily. In ECN, the cooperation performance is evaluated by means of a pervasive metrics termed as QoC based on the ECN cooperative mode preferences. Further, the ECN performance is improved by means of a cooperative optimization algorithm designed in this paper. The efficiency of the framework is proved in the experimental section based on the wearable medical sensor dataset. The edge cooperative optimization algorithm feasibility is further verified based on a case study. Future scope is directed towards expanding the datasets and further fine tuning the efficiency of the framework. 
Journal of Ubiquitous Computing and Communication Technologies (UCCT) (2021)

Vol.03/ No.01

Pages: $33-42$

https://www.irojournals.com/jucct/

DOI: https://doi.org/10.36548/jucct.2021.1.004

\section{References}

[1] Rahman, M. A., Hossain, M. S., Loukas, G., Hassanain, E., Rahman, S. S., Alhamid, M. F., \& Guizani, M. (2018). Blockchain-based mobile edge computing framework for secure therapy applications. IEEE Access, 6, 72469-72478.

[2] Sodhro, A. H., Obaidat, M. S., Abbasi, Q. H., Pace, P., Pirbhulal, S., Fortino, G., ... \& Qaraqe, M. (2019). Quality of service optimization in an iot-driven intelligent transportation system. IEEE Wireless Communications, 26(6), 10-17.

[3] Sodhro, A. H., Luo, Z., Sangaiah, A. K., \& Baik, S. W. (2019). Mobile edge computing based QoS optimization in medical healthcare applications. International Journal of Information Management, 45, 308-318.

[4] Wan, L., Sun, L., Kong, X., Yuan, Y., Sun, K., \& Xia, F. (2019). Task-driven resource assignment in mobile edge computing exploiting evolutionary computation. IEEE Wireless Communications, 26(6), 94-101.

[5] Sodhro, A. H., Pirbhulal, S., \& De Albuquerque, V. H. C. (2019). Artificial intelligencedriven mechanism for edge computing-based industrial applications. IEEE Transactions on Industrial Informatics, 15(7), 4235-4243.

[6] Ren, J., Wang, H., Hou, T., Zheng, S., \& Tang, C. (2019). Federated learning-based computation offloading optimization in edge computing-supported internet of things. IEEE Access, 7, 69194-69201.

[7] Vimal, S., Khari, M., Dey, N., Crespo, R. G., \& Robinson, Y. H. (2020). Enhanced resource allocation in mobile edge computing using reinforcement learning based MOACO algorithm for IIOT. Computer Communications, 151, 355-364.

[8] Alam, M. G. R., Munir, M. S., Uddin, M. Z., Alam, M. S., Dang, T. N., \& Hong, C. S. (2019). Edge-of-things computing framework for cost-effective provisioning of healthcare data. Journal of Parallel and Distributed Computing, 123, 54-60.

[9] Oueida, S., Kotb, Y., Aloqaily, M., Jararweh, Y., \& Baker, T. (2018). An edge computing based smart healthcare framework for resource management. Sensors, 18(12), 4307.

[10] Abbasi, M., Mohammadi-Pasand, E., \& Khosravi, M. R. (2021). Intelligent workload allocation in IoT-Fog-cloud architecture towards mobile edge computing. Computer Communications, 169, 71-80.

[11] Rahman, M. A., \& Hossain, M. S. (2021). An Internet of medical things-enabled edge computing framework for tackling COVID-19. IEEE Internet of Things Journal. 
Journal of Ubiquitous Computing and Communication Technologies (UCCT) (2021)

Vol.03/ No.01

Pages: $33-42$

https://www.irojournals.com/jucct/

DOI: https://doi.org/10.36548/jucct.2021.1.004

[12] Bhalaji, N. (2020). Efficient and secure data utilization in mobile edge computing by data replication. Journal of ISMAC, 2(01), 1-12.

[13] Sivaganesan, D. (2019). Design and development ai-enabled edge computing for intelligent-iot applications. Journal of trends in Computer Science and Smart technology (TCSST), 1(02), 84-94.

[14] Bhalaji, N. (2020). Reliable Data Transmission with Heightened Confidentiality and Integrity in IOT Empowered Mobile Networks. Journal of ISMAC, 2(02), 106-117.

[15] Smys, S., \& Ranganathan, G. (2020). Performance Evaluation of Game Theory Based Efficient Task Scheduling For Edge Computing. Journal of ISMAC, 2(01), 50-61. 